Canad. J. Math. Vol. 63 (6), 2011 pp. 1328-1344

doi:10.4153/CJM-2011-034-2

(C) Canadian Mathematical Society 2011

\title{
On a Conjecture of Chowla and Milnor
}

\author{
Sanoli Gun, M. Ram Murty, and Purusottam Rath
}

Abstract. In this paper, we investigate a conjecture due to $\mathrm{S}$. and $\mathrm{P}$. Chowla and its generalization by Milnor. These are related to the delicate question of non-vanishing of $L$-functions associated to periodic functions at integers greater than 1 . We report on some progress in relation to these conjectures. In a different vein, we link them to a conjecture of Zagier on multiple zeta values and also to linear independence of polylogarithms.

\section{Introduction}

Let $q>1$ be an integer and $f$ be a periodic arithmetic function with period $q$. For a complex number $s \in \mathbf{C}$ with $\operatorname{Re}(s)>1$, consider the Dirichlet series

$$
\mathrm{L}(s, f)=\sum_{n=1}^{\infty} \frac{f(n)}{n^{s}} .
$$

This is called the L-function associated to $f$. For a real number $x$ with $0<x \leqslant 1$ and a complex number $s \in \mathbf{C}$ with $\operatorname{Re}(s)>1$, one defines the Hurwitz zeta function as

$$
\zeta(s, x)=\sum_{n=0}^{\infty} \frac{1}{(n+x)^{s}}
$$

Hurwitz proved that $\zeta(s, x)$ extends analytically to the entire complex plane, apart from $s=1$, where it has a simple pole with residue 1 . Note that for $x=1$, Hurwitz zeta function is the classical Riemann zeta function. Since

$$
\mathrm{L}(s, f)=q^{-s} \sum_{a=1}^{q} f(a) \zeta(s, a / q),
$$

$\mathrm{L}(s, f)$ extends meromorphically to the complex plane with a possible simple pole at $s=1$ with residue $q^{-1} \sum_{a=1}^{q} f(a)$.

In this paper, we investigate the algebraic nature and non-vanishing of the numbers $\mathrm{L}(k, f)$ for integers $k>1$ when $f$ takes rational values. The simplest example is the Riemann zeta function, whose transcendence at even integers is well known while its values at odd integers are mysterious. However, for an arbitrary rationalvalued function $f$, even the non-vanishing of $\mathrm{L}(k, f)$ is not assured and has deep significance.

Received by the editors January 12, 2010; revised April 28, 2010.

Published electronically June 20, 2011.

AMS subject classification: 11F20, 11F11. 
The non-vanishing as well as the transcendental nature of $L(1, f)$ when $f$ is rational or even algebraic valued have been investigated in [3] and [1]. In the recent works [6] and [7], the transcendental nature of Hecke $L$-functions at $s=1$ has been carried out.

The motivation for our present work germinates from the following seemingly innocuous question, asked by P. and S. Chowla [4].

Conjecture (Chowla-Chowla) Let $p$ be any prime and $f$ be any rational-valued periodic function with period $p$. Then $\mathrm{L}(2, f) \neq 0$ except in the case when

$$
f(1)=f(2)=\cdots=f(p-1)=\frac{f(p)}{1-p^{2}} .
$$

Milnor [9] interpreted the above conjecture in terms of the linear independence of the Hurwitz zeta function and generalized it for all $k>1$. Substituting

$$
\left(p^{k}-1\right) \zeta(k)=\sum_{a=1}^{p-1} \zeta(k, a / p)
$$

in the expression (1), we have

$$
\mathrm{L}(k, f)=p^{-k} \sum_{a=1}^{p-1}\left[f(a)+\frac{f(p)}{\left(p^{k}-1\right)}\right] \zeta(k, a / p) .
$$

Thus the Chowla-Chowla conjecture is clearly equivalent to the following conjecture for $k=2$.

Conjecture (Milnor) For any integer $k>1$, the real numbers

$$
\zeta(k, 1 / p), \zeta(k, 2 / p), \ldots, \zeta(k,(p-1) / p)
$$

are linearly independent over $\mathbf{Q}$.

Milnor's conjecture appears more natural and puts the Chowla-Chowla conjecture in perspective. Further, for $q$ not necessarily prime, Milnor suggested the following generalisation of the Chowla conjecture.

Conjecture (Chowla-Milnor) Let $k>1, q>2$ be integers. Then the following $\varphi(q)$ real numbers are linearly independent over $\mathbf{Q}$ :

$$
\zeta(k, a / q) \quad \text { with }(a, q)=1,1 \leq a \leq q .
$$

In relation to the Chowla-Milnor conjecture, we define the following Q-linear spaces.

Definition 1 For any integer $k>1$, the Chowla-Milnor space is the Q-linear space $\mathrm{V}_{k}(q)$ defined by

$$
\mathrm{V}_{k}(q)=\mathbf{Q}-\text { Span of }\{\zeta(k, a / q): 1 \leq a<q,(a, q)=1\}
$$


The Chowla-Milnor conjecture asserts that the dimension of $V_{k}(q)$ for $k>1$ is $\varphi(q)$. The dimensions of the Chowla-Milnor spaces also hold the key to the understanding of Riemann zeta values at odd positive integers. In Section 1, we derive the following non-trivial lower bound for their dimension.

Theorem 1 Let $k>1$ and $q>2$, then

$$
\operatorname{dim}_{\mathrm{Q}} \mathrm{V}_{k}(q) \geq \frac{\varphi(q)}{2}
$$

Any improvement of the above bound will have remarkable consequences. For instance, any improvement of the above lower bound for odd $k=2 d+1$ would establish the irrationality of numbers of the form $\zeta(2 d+1) / \pi^{2 d+1}$ for $d \geqslant 1$. In this connection, we prove the following theorem.

Theorem 2 Let $k>1$ be an odd integer and $q>2$ and $r>2$ be two co-prime integers. Then either

$$
\operatorname{dim}_{\mathbf{Q}} \mathrm{V}_{k}(q) \geq \frac{\varphi(q)}{2}+1
$$

or

$$
\operatorname{dim}_{\mathbf{Q}} \mathrm{V}_{k}(r) \geq \frac{\varphi(r)}{2}+1
$$

Thus in particular, there exists $q_{0}$ such that

$$
\operatorname{dim}_{\mathbf{Q}} \mathrm{V}_{k}(q) \geq \frac{\varphi(q)}{2}+1
$$

for any $q$ co-prime to $q_{0}$.

In Section 2, we establish a link between the Chowla-Milnor conjecture and the multiple zeta values (MZVs).

Definition 2 Let $l, s_{1}, \ldots, s_{l}$ be positive integers with $s_{1}>1$. Then the multiple zeta values (MZVs) are defined as

$$
\zeta\left(s_{1}, \ldots, s_{l}\right)=\sum_{n_{1}>\cdots>n_{l} \geq 1} \frac{1}{n_{1}^{s_{1}}} \cdots \frac{1}{n_{l}^{s_{l}}} .
$$

When $l=1$, we get the classical zeta values. The sum $s_{1}+\cdots+s_{l}$ is called the weight while $l$ is called the length of $\zeta\left(s_{1}, \ldots, s_{l}\right)$.

Definition 3 Let $k \geqslant 2$ be an integer. Then $\mathrm{W}_{k}$ is the $\mathrm{Q}$-linear space spanned by all $\zeta\left(s_{1}, \ldots, s_{l}\right)$ with integers $l \geqslant 1, s_{1}>1$ such that $s_{1}+\cdots+s_{l}=k$.

We have the following interesting conjecture due to Zagier [14] about the dimension of $\mathrm{W}_{k}$. 
Conjecture (Zagier) The dimension $d_{k}$ of the space $\mathrm{W}_{k}$ for $k \geq 3$ is given by the recurrence

$$
\delta_{k}=\delta_{k-2}+\delta_{k-3}
$$

with $\delta_{0}=1, \delta_{1}=0$ and $\delta_{2}=1$.

It is now known, thanks to the works of Goncharov [5] and Terasoma [13], that $d_{k} \leq \delta_{k}$. The only known cases of Zagier's conjecture are $d_{2}=d_{3}=d_{4}=1$. Thus we do not have a single example where the dimension of $\mathrm{W}_{k}$ is at least 2 . In this connection, we prove the following result.

Theorem 3 The Chowla-Milnor conjecture implies that the dimension of $\mathrm{W}_{4 d+2}$ is at least 2 for all $d \geqslant 1$.

Thus, in particular, the Chowla-Milnor conjecture shows that there is an infinite family of $\mathrm{W}_{k}$ 's with dimension at least 2 .

In Section 3, we investigate the Chowla-Milnor conjecture in terms of linear independence of polylogarithms.

Definition 4 For an integer $k \geqslant 2$ and complex numbers $z \in \mathbf{C}$ with $|z| \leqslant 1$, the polylogarithm function $\operatorname{Li}_{k}(z)$ is defined by

$$
\operatorname{Li}_{k}(z)=\sum_{n=1}^{\infty} \frac{z^{n}}{n^{k}} .
$$

For $k=1$, the series is $-\log (1-z)$, provided that $|z|<1$. Analogous to Baker's theorem on linear forms in logarithms, we may have the following conjecture about polylogarithms.

Conjecture (Polylog) Suppose that $\alpha_{1}, \ldots, \alpha_{n}$ are algebraic numbers $\left|\alpha_{i}\right| \leqslant 1$ such that $\operatorname{Li}_{k}\left(\alpha_{1}\right), \ldots, \operatorname{Li}_{k}\left(\alpha_{n}\right)$ are linearly independent over $\mathbf{Q}$. Then they are linearly independent over the field of algebraic numbers $\overline{\mathbf{Q}}$.

Apart from the case $k=1$, which is a special case of Baker's theorem, almost nothing is known about the above conjecture. We prove the following theorem using a method developed by Baker, Birch, and Wirsing.

Theorem 4 Assume that the polylog conjecture is true. Then the Chowla-Milnor conjecture is true for all $q>1$ and $k>1$.

In the final section, we derive some interesting consequences of the ChowlaMilnor conjecture. We also formulate a stronger version of it that appears natural to us. We note that this is related to some long-standing conjectures in number theory.

\section{Proofs of Theorems 1 and 2}

The crucial ingredient for the proof of Theorem 1 is the following lemma due to Okada [12] about the linear independence of co-tangent values at rational arguments. 
Lemma 1 Let $k$ and $q$ be positive integers with $k>0$ and $q>2$. Let $\mathrm{T}$ be a set of $\varphi(q) / 2$ representations $\bmod q$ such that the union $\mathrm{T} \cup(-\mathrm{T})$ constitutes a complete set of co-prime residue classes mod $q$. Then the set of real numbers

$$
\left.\frac{d^{k-1}}{d z^{k-1}} \cot (\pi z)\right|_{z=a / q}, \quad a \in T
$$

is linearly independent over $\mathbf{Q}$.

Proof of Theorem 1 To start with, we note that the space $V_{k}(q)$ is also spanned by the following sets of real numbers:

$$
\begin{array}{r}
\{\zeta(k, a / q)+\zeta(k, 1-a / q) \mid(a, q)=1,1 \leq a<q / 2\}, \\
\{\zeta(k, a / q)-\zeta(k, 1-a / q) \mid(a, q)=1,1 \leq a<q / 2\} .
\end{array}
$$

We define the following $\mathbf{Q}$-linear subspaces of $\mathrm{V}_{k}(q)$ :

$$
\begin{aligned}
\mathrm{V}_{k}(q)^{+} & =\mathbf{Q}-\operatorname{span} \text { of }\{\zeta(k, a / q)+\zeta(k, 1-a / q) \mid(a, q)=1,1 \leq a<q / 2\}, \\
\mathrm{V}_{k}(q)^{-} & =\mathbf{Q}-\operatorname{span} \text { of }\{\zeta(k, a / q)-\zeta(k, 1-a / q) \mid(a, q)=1,1 \leq a<q / 2\} .
\end{aligned}
$$

Then we have the following (see [11], for instance)

$$
\zeta(k, a / q)+(-1)^{k} \zeta(k, 1-a / q)=\left.\frac{(-1)^{k-1}}{(k-1) !} D^{k-1}(\pi \cot \pi z)\right|_{z=a / q}
$$

where $D^{k}=\frac{d^{k}}{d z^{k}}$. Applying the above lemma, we see that

$$
\operatorname{dim}_{\mathrm{Q}} \mathrm{V}_{k}(q) \geq \frac{\varphi(q)}{2} .
$$

This completes the proof of Theorem 1.

For the proof of Theorem 2, we shall need the following proposition, which is of independent interest.

Proposition 1 Let $k>1$ be an odd integer. For $q \geq 3$, let $\mathrm{Z}_{k}(a, q)$ for any $(a, q)=1$ and $1 \leq a<q / 2$ be the number

$$
\mathrm{Z}_{k}(a, q):=\frac{\zeta(k, a / q)-\zeta(k, 1-a / q)}{(2 \pi i)^{k}} .
$$

If $\varphi(q) \geq 4$, then the numbers

$$
\frac{\mathrm{Z}_{k}(a, q)}{i}
$$

are all real irrational algebraic numbers. 
Proof For any odd periodic function $f$ with period $q$, we have (see [11])

$$
2 \mathrm{~L}(k, f)=\frac{(2 \pi i)^{k}}{k !} \sum_{a=1}^{q} \widehat{f}(a) \mathrm{B}_{k}(a / q),
$$

where

$$
\mathrm{B}_{k}(x)=\frac{-k !}{(2 \pi i)^{k}} \sum_{\substack{n=-\infty \\ n \neq 0}}^{\infty} \frac{e^{2 \pi i n x}}{n^{k}}
$$

is the $k$-th Bernoulli polynomial and

$$
\widehat{f}(n):=\frac{1}{q} \sum_{b=1}^{q} f(b) e^{2 \pi i b n / q}
$$

is the Fourier transform of $f$. For $a$ co-prime to $q$, we consider the following odd function

$$
\delta_{a}(n)= \begin{cases}1, & \text { if } n=a \\ -1, & \text { if } n=q-a \\ 0, & \text { otherwise. }\end{cases}
$$

Then

$$
2 \mathrm{~L}\left(k, \delta_{a}\right)=\frac{(2 \pi i)^{k}}{k !} \sum_{b=1}^{q} \widehat{\delta}_{a}(b) \mathrm{B}_{k}(b / q),
$$

where $\widehat{\delta}_{a}(n)=\frac{1}{q} \sum_{b=1}^{q} \delta_{a}(b) e^{\frac{2 i \pi b n}{q}}=\frac{1}{q}\left[\zeta_{q}^{a n}-\zeta_{q}^{-a n}\right]$, where $\zeta_{q}=e^{\frac{2 \pi i}{q}}$. Since

$$
\mathrm{L}\left(k, \delta_{a}\right)=\frac{1}{q^{k}} \sum_{b=1}^{q} \delta_{a}(b) \zeta(k, b / q)=\frac{1}{q^{k}}[\zeta(k, a / q)-\zeta(k, 1-a / q)],
$$

we have

$$
\frac{\zeta(k, a / q)-\zeta(k, 1-a / q)}{(2 \pi i)^{k}}=\frac{q^{k-1}}{2 k !} \sum_{b=1}^{q}\left(\zeta_{q}^{a b}-\zeta_{q}^{-a b}\right) \mathrm{B}_{k}(b / q) .
$$

Thus we have

$$
\mathrm{Z}_{k}(a, q)=\frac{q^{k-1}}{2 k !} \sum_{b=1}^{q}\left(\zeta_{q}^{a b}-\zeta_{q}^{-a b}\right) \mathrm{B}_{k}(b / q)
$$

Clearly,

$$
\mathrm{Z}_{k}(a, q) \in \mathbf{Q}\left(\zeta_{q}\right)
$$

We have

$$
\begin{gathered}
\zeta(k, a / q)-\zeta(k, 1-a / q)=\left.\frac{1}{(k-1) !} \mathrm{D}^{k-1}(\pi \cot \pi z)\right|_{z=a / q}, \\
\frac{Z_{k}(a, q)}{i}= \pm \frac{\zeta(k, a / q)-\zeta(k, 1-a / q)}{(2 \pi)^{k}} .
\end{gathered}
$$


Thus, by Lemma 1, the set of real algebraic numbers

$$
\frac{\mathrm{Z}_{k}(a, q)}{i} \text { with }(a, q)=1,1 \leq a<q / 2
$$

is linearly independent over the rationals.

Let $\mathrm{G}$ be the Galois group of $\mathbf{Q}\left(\zeta_{q}\right)$ over $\mathbf{Q}$ which is given by the maps

$$
\sigma_{c}\left(\zeta_{q}\right)=\zeta_{q}^{c}
$$

where $c$ runs over the co-prime residue classes modulo $q$. We see immediately that

$$
\sigma_{c}\left(\mathrm{Z}_{k}(a, q)\right)=\mathrm{Z}_{k}(a c, q) .
$$

Thus the set of algebraic numbers

$$
\mathrm{Z}_{k}(a, q) \quad \text { with }(a, q)=1,1 \leq a<q / 2
$$

are all conjugates of each other. Since they are linearly independent over $\mathbf{Q}$ and since $\varphi(q) \geqslant 4$, all the numbers in equation (3) are necessarily irrational.

Thus, we have the following bound

$$
\frac{\varphi(q)}{2} \leq \operatorname{dim}_{\mathrm{Q}} \mathrm{V}_{k}(q) \leq \varphi(q)
$$

We now proceed to prove Theorem 2 which improves the lower bound for infinitely many $q$.

Proof of Theorem 2 Let $q$ and $r$ be two co-prime integers. Suppose that

$$
\operatorname{dim}_{\mathbf{Q}} \mathrm{V}_{k}(q)=\frac{\varphi(q)}{2} .
$$

Then the numbers

$$
\zeta(k, a / q)-\zeta(k, 1-a / q), \quad \text { where }(a, q)=1,1 \leq a<q / 2
$$

generate $\mathrm{V}_{k}(q)$. Now

$$
q^{k} \zeta(k) \prod_{p \mid q}\left(1-p^{-k}\right)=\sum_{\substack{a=1 \\(a, q)=1}}^{q-1} \zeta(k, a / q) \in \mathrm{V}_{k}(q) .
$$

and hence

$$
\begin{aligned}
\zeta(k) & =\sum_{\substack{(a, q)=1 \\
1 \leq a<q / 2}} \lambda_{a}[\zeta(k, a / q)-\zeta(k, 1-a / q)], \lambda_{a} \in \mathbf{Q} \\
& =(2 \pi i)^{k} \sum_{\substack{(a, q)=1 \\
1 \leq a<q / 2}} \mathrm{Z}_{k}(a, q) \lambda_{a}, \lambda_{a} \in \mathbf{Q} .
\end{aligned}
$$


Thus by Proposition 1, we have

$$
\frac{\zeta(k)}{i \pi^{k}} \in \mathbf{Q}\left(\zeta_{q}\right)
$$

Similarly, if

$$
\operatorname{dim}_{\mathbf{Q}} \mathrm{V}_{k}(r)=\frac{\varphi(r)}{2}
$$

then

$$
\frac{\zeta(k)}{i \pi^{k}} \in \mathbf{Q}\left(\zeta_{r}\right)
$$

and hence

$$
\frac{\zeta(k)}{i \pi^{k}} \in \mathbf{Q}\left(\zeta_{q}\right) \cap \mathbf{Q}\left(\zeta_{r}\right) .
$$

Since $q$ and $r$ are co-prime, $\mathbf{Q}\left(\zeta_{q}\right) \cap \mathbf{Q}\left(\zeta_{r}\right)=\mathbf{Q}$ and hence we arrive at a contradiction. Thus

$$
\operatorname{dim}_{\mathbf{Q}} \mathrm{V}_{k}(q)>\frac{\varphi(q)}{2} \text { or } \quad \operatorname{dim}_{\mathbf{Q}} \mathrm{V}_{k}(r)>\frac{\varphi(r)}{2}
$$

We have the following immediate corollary.

Corollary 1 Let $k$ be an odd integer. Then $\operatorname{dim}_{\mathrm{Q}} \mathrm{V}_{k}(3)=2$ or $\operatorname{dim}_{\mathrm{Q}} \mathrm{V}_{k}(4)=2$.

This should be compared with Proposition 7 in the last section.

We now consider the case when $k$ is an even integer. Here, we have the following proposition.

Proposition 2 Let $k>1$ be an even integer. For $q \geq 3$, let $\mathrm{Z}_{k}(a, q)$ for any $(a, q)=1$ and $1 \leq a<q / 2$ be the number

$$
\mathrm{Z}_{k}(a, q):=\frac{\zeta(k, a / q)+\zeta(k, 1-a / q)}{(2 \pi i)^{k}}
$$

If $\varphi(q) \geq 4$, then the $Z_{k}(a, q)$ 's are all irrational real numbers lying in $\mathbf{Q}\left(\zeta_{q}\right)$. Further,

$$
\sum_{\substack{a=1 \\(a, q)=1}}^{q / 2} c_{a} \mathrm{Z}_{k}(a, q) \in \mathbf{Q}, c_{a} \in \mathbf{Q}
$$

if and only if all the $c_{a}$ 's are equal.

Proof The proof of first part follows exactly as the proof of Proposition 1. For the last assertion, suppose that

$$
\sum_{\substack{a=1 \\(a, q)=1}}^{q / 2} c_{a} \mathrm{Z}_{k}(a, q)=\alpha
$$


where $c_{a}, \alpha \in \mathbf{Q}$. We note that $(\mathbf{Z} / q \mathbf{Z})^{\times} / \pm 1$ acts transitively on the set

$$
\left\{\mathrm{Z}_{k}(a, q): 1 \leq a<q / 2,(a, q)=1\right\}
$$

Since the set

$$
\left\{Z_{k}(a, q): 1 \leq a<q / 2,(a, q)=1\right\}
$$

is linearly independent over $\mathbf{Q}$, we see that all the $c_{a}$ 's are necessarily equal. The converse is also true as

$$
\sum_{\substack{a=1 \\(a, q)=1}}^{q} \zeta(k, a / q)
$$

is a rational multiple of $\zeta(k)$.

We have the following immediate corollary.

Corollary 2 Let $k>1$ be an even integer and $\chi$ be a non-trivial even quadratic character modulo q. Then

$$
\frac{\mathrm{L}(k, \chi)}{\pi^{k}} \in \mathbf{Q}\left(\zeta_{q}\right) \backslash \mathbf{Q} .
$$

Proof Let $\chi$ be a non-trivial quadratic character modulo $q$ which is even. For such a character, we have

$$
\begin{aligned}
\mathrm{L}(k, \chi) & =\sum_{a=1}^{q} \chi(a) \zeta(k, a / q) \\
& =\sum_{a=1}^{q / 2} \chi(a)[\zeta(k, a / q)+\zeta(k, 1-a / q)] \\
& =(2 \pi i)^{k} \sum_{a=1}^{q / 2} \chi(a) \mathrm{Z}_{k}(a, q) .
\end{aligned}
$$

Hence the assertion.

We note that for $k$ even, $\pi^{-k} \mathrm{~L}(k, \chi)$ is rational when $\chi$ is the trivial character.

\section{Proof of Theorem 3}

We shall need the following lemma for the proof of Theorem 3.

Lemma 2 If

$$
\left[\frac{\zeta(2 k+1)}{\pi^{2 k+1}}\right]^{2}
$$

is not a rational number, then $d_{4 k+2} \geq 2$. 
Proof We have

$$
\zeta\left(s_{1}\right) \zeta\left(s_{2}\right)=\zeta\left(s_{1}, s_{2}\right)+\zeta\left(s_{2}, s_{1}\right)+\zeta\left(s_{1}+s_{2}\right) .
$$

Thus

$$
\zeta(2 k+1)^{2}=2 \zeta(2 k+1,2 k+1)+\zeta(4 k+2) .
$$

Since

$$
\left[\frac{\zeta(2 k+1)}{\pi^{2 k+1}}\right]^{2} \notin \mathbf{Q} \quad \text { and } \quad \frac{\zeta(4 k+2)}{\pi^{4 k+2}} \in \mathbf{Q},
$$

we see that $\zeta(2 k+1,2 k+1)$ is not in the $\mathbf{Q}$-span of $\zeta(4 k+2)$ and hence the dimension of the space $\mathrm{W}_{4 k+2}$ is at least 2 .

We shall also need the following proposition for proof of Theorem 3.

Proposition 3 Suppose that the Chowla-Milnor conjecture is true. Then

$$
\left[\frac{\zeta(2 d+1)}{\pi^{2 d+1}}\right]^{2}
$$

is irrational for all $d \geqslant 1$.

Proof We require a few generalities on the theory of Gauss sums associated with quadratic characters. Let $\Delta<0$ be a fundamental discriminant. Then the Kronecker symbol

$$
\chi(n)=\left(\frac{\Delta}{n}\right)
$$

is an odd, primitive, quadratic character modulo $|\Delta|$ (see [10, p. 297]). Let $q=|\Delta|$.

For each such character $\chi$, the associated Gauss sum $\tau(\chi)$ is given by $[10$, Theorem 9.17, p. 300]:

$$
\tau(\chi)=\sum_{a=1}^{q} \chi(a) \zeta_{q}^{a}=i \sqrt{q} .
$$

Further, by primitivity of $\chi$, we have:

$$
\tau(\chi, b)=\sum_{a=1}^{q} \chi(a) \zeta_{q}^{a b}=\bar{\chi}(b) \tau(\chi)=\bar{\chi}(b) i \sqrt{q} .
$$

Since $\chi$ is odd, we have

$$
\sum_{a=1}^{q / 2} \chi(a)\left(\zeta_{q}^{a b}-\zeta_{q}^{-a b}\right)=\bar{\chi}(b) i \sqrt{q}
$$

We multiply both sides by $\mathrm{B}_{2 d+1}(b / q)$ and sum over $b=1$ to $q$ to get

$$
\sum_{a=1}^{q / 2} \chi(a) \sum_{b=1}^{q}\left(\zeta_{q}^{a b}-\zeta_{q}^{-a b}\right) \mathrm{B}_{2 d+1}(b / q)=i \sqrt{q} \sum_{b=1}^{q} \bar{\chi}(b) \mathrm{B}_{2 d+1}(b / q) .
$$


Let $k=2 d+1$. Recall that for any such odd $k$ and $q>2$, as mentioned in the proof of Proposition 1, we have

$$
\frac{\zeta(k, a / q)-\zeta(k, 1-a / q)}{(2 \pi i)^{k}}=\frac{q^{k-1}}{2 k !} \sum_{b=1}^{q}\left(\zeta_{q}^{a b}-\zeta_{q}^{-a b}\right) \mathrm{B}_{k}(b / q)
$$

for any $(a, q)=1$ and $1 \leq a<q / 2$. Thus the number $i \sqrt{q}$ lies in the $\mathbf{Q}$-linear space generated by the real numbers

$$
\frac{\zeta(k, a / q)-\zeta(k, 1-a / q)}{(2 \pi i)^{k}} \text { with }(a, q)=1,1 \leq a<q / 2 .
$$

On the other hand, since $\zeta(k)$ is a rational multiple of

$$
\sum_{\substack{a=1 \\(a, q)=1}}^{q / 2}(\zeta(k, a / q)+\zeta(k, 1-a / q))
$$

we see that $\zeta(k) /(2 \pi i)^{k}$ lies in the subspace generated by

$$
\frac{\zeta(k, a / q)+\zeta(k, 1-a / q)}{(2 \pi i)^{k}} \text { with }(a, q)=1,1 \leq a<q / 2 .
$$

Thus the Chowla-Milnor conjecture for the modulus $q$ implies that $\zeta(k) /(2 \pi i)^{k}$ and $i \sqrt{q}$ lie in disjoint $\mathbf{Q}$-spaces. Thus their ratio is irrational and hence for any such $q$, we have

$$
\frac{\zeta(2 d+1)}{\pi^{2 d+1} \sqrt{q}} \notin \mathbf{Q} .
$$

Thus, if the Chowla-Milnor conjecture is true for all modulus, then

$$
\left[\frac{\zeta(2 d+1)}{\pi^{2 d+1}}\right]^{2}
$$

is irrational for all $d \geqslant 1$.

Finally, we give a proof of Theorem 3.

Proof of Theorem 3 Now suppose that that the dimension of the space $\mathrm{W}_{4 d+2}$ is 1 . Then by Lemma 2, we have

$$
\frac{\zeta(2 d+1)}{\pi^{2 d+1}}=r \sqrt{q}, \quad \text { where } q \in \mathbf{N}, r \in \mathbf{Q} .
$$

But then by Proposition 3, the Chowla-Milnor conjecture is false for the modulus $q$. 
On a Conjecture of Chowla and Milnor

\section{Proof of Theorem 4}

For the proof of Theorem 4, we shall need the following lemma (see [8, p. 548]).

Lemma 3 Let $G$ be any finite abelian group of order $n$ and $F: G \rightarrow \mathbf{C}$ be any complexvalued function on $G$. The determinant of the $n \times n$ matrix given by $\left(F\left(x y^{-1}\right)\right)$ as $x, y$ range over the group elements is called the Dedekind determinant and is equal to

$$
\prod_{\chi}\left(\sum_{x \in G} \chi(x) F(x)\right)
$$

where the product is over all characters $\chi$ of $G$.

We shall now give a proof of Theorem 4 following the methodology employed in [1].

Proof of Theorem 4 Let $k, q>1$ be integers. For simplicity, we prove the theorem when $q=p$ is a prime. The proof of the general case is identical.

Let $f$ be a rational-valued periodic function with prime period $p$ and suppose that $\mathrm{L}(k, f)=0$. As before, let

$$
\widehat{f}(n)=\sum_{a=1}^{p} f(a) \zeta_{p}^{-a n}
$$

and by Fourier inversion

$$
f(n)=\sum_{a=1}^{p} \widehat{f}(a) \zeta_{p}^{a n}
$$

Then

$$
\mathrm{L}(k, f)=\sum_{n=1}^{\infty} \frac{f(n)}{n^{k}}=\sum_{n=1}^{\infty} \frac{1}{n^{k}} \sum_{a=1}^{p} \widehat{f}(a) \zeta_{p}^{a n}
$$

and hence

$$
\mathrm{L}(k, f)=\sum_{a=1}^{p} \widehat{f}(a) \operatorname{Li}_{k}\left(\zeta_{p}^{a}\right)=0 .
$$

Let

$$
\operatorname{Li}_{k}\left(\alpha_{1}\right), \ldots, \operatorname{Li}_{k}\left(\alpha_{t}\right)
$$

be a maximal Q-linear independent subset of

$$
\left\{\operatorname{Li}_{k}\left(\zeta_{p}^{a}\right) \mid 1 \leq a \leq p-1\right\} .
$$

Let

$$
\operatorname{Li}_{k}\left(\zeta_{p}^{a}\right)=\sum_{b=1}^{t} \mathrm{~A}_{a b} \operatorname{Li}_{k}\left(\alpha_{b}\right)
$$

where $\mathrm{A}_{a b}$ are rational numbers. Then

$$
\beta_{1} \operatorname{Li}_{k}\left(\alpha_{1}\right)+\cdots+\beta_{t} \operatorname{Li}_{k}\left(\alpha_{t}\right)=0
$$


where

$$
\beta_{b}=\sum_{a=1}^{p} \widehat{f}(a) \mathrm{A}_{a b} .
$$

Since $f$ is rational valued, $\widehat{f}$ is algebraic valued. Thus by the polylog conjecture, we have

$$
\beta_{b}=\sum_{a=1}^{p} \widehat{f}(a) \mathrm{A}_{a b}=0, \quad 1 \leq b \leq t .
$$

Thus for any automorphism $\sigma$ of the field $\overline{\mathbf{Q}}$ over $\mathbf{Q}$, we have

$$
\sum_{a=1}^{p} \sigma(\widehat{f}(a)) \mathrm{A}_{a b}=0, \quad 1 \leq b \leq t
$$

and hence

$$
\sum_{a=1}^{p} \sigma(\widehat{f}(a)) \operatorname{Li}_{k}\left(\zeta_{p}^{a}\right)=0
$$

In particular, if for $1 \leq h \leq p-1, \sigma_{h}$ is the element of the Galois group of $\mathbf{Q}\left(\zeta_{p}\right)$ over $\mathbf{Q}$ such that

$$
\sigma_{h}\left(\zeta_{p}\right)=\zeta_{p}^{h}
$$

we have,

$$
\sigma(\widehat{f}(n))=\widehat{f}_{h}(n)
$$

where

$$
f_{h}(n)=f\left(n h^{-1}\right) .
$$

Thus, we have

$$
\mathrm{L}\left(k, f_{h}\right)=\sum_{n=1}^{\infty} \frac{f_{h}(n)}{n^{k}}=0
$$

for all $1 \leq h \leq p-1$. This gives that

$$
\mathrm{L}\left(k, f_{h}\right)=p^{-k} \sum_{a=1}^{p-1}\left[f_{h}(a)+\frac{f_{h}(p)}{\left(p^{k}-1\right)}\right] \zeta(k, a / p)=0
$$

for all $1 \leq h \leq p-1$.

Now, making a change of variable and noting that $f_{h}(p)=f(p)$, we have

$$
\mathrm{L}\left(k, f_{h}\right)=p^{-k} \sum_{a=1}^{p-1}\left[f(a)+\frac{f(p)}{\left(p^{k}-1\right)}\right] \zeta(k, a h / p)=0
$$

for all $1 \leq h \leq p-1$. We treat this as a matrix equation with B being the $(p-1) \times$ $(p-1)$ matrix whose $(a, h)$-th entry is given by $\zeta(k, a h / p)$. Then by the evaluation of the Dedekind determinant as in Lemma 3, we have

$$
\operatorname{Det}(\mathrm{B})= \pm \prod_{\chi} p^{k} L(k, \chi) \neq 0 .
$$


Thus the matrix $B$ is invertible and hence by the equation (4), we have

$$
f(a)+\frac{f(p)}{\left(p^{k}-1\right)}=0, \quad 1 \leq a \leq p-1
$$

and hence

$$
f(1)=f(2)=\cdots=\frac{f(p)}{\left(1-p^{k}\right)} .
$$

Thus the proof of the theorem for the case when the modulus is a prime. The proof for an arbitrary modulus $q$ is identical, the final Dedekind determinant being associated to the group $(\mathbf{Z} / q \mathbf{Z})^{\times}$and of size $\varphi(q) \times \varphi(q)$.

\section{Concluding Remarks}

We begin the section by mentioning some interesting consequences of the ChowlaMilnor conjecture.

Proposition 4 The Chowla-Milnor conjecture for the single modulus $q=4$ is equivalent to the irrationality of $\zeta(2 d+1) / \pi^{2 d+1}$ for all $d \geq 1$.

Proof Let $k=2 d+1$. We note that $\mathrm{V}_{k}(4)$ is generated by $\zeta(k, 1 / 4)$ and $\zeta(k, 3 / 4)$ and

$$
\left(4^{k}-2^{k}\right) \zeta(k)=\zeta(k, 1 / 4)+\zeta(k, 3 / 4)
$$

Also,

$$
\zeta(k, 1 / 4)-\zeta(k, 3 / 4)=\left.\frac{1}{(k-1) !} D^{k-1}(\pi \cot \pi z)\right|_{z=1 / 4} .
$$

But $\left.D^{k-1}(\pi \cot \pi z)\right|_{z=1 / 4}$ is a rational multiple of $\pi^{k}$. Therefore the rationality of $\zeta(k) / \pi^{k}$ will imply that $\mathrm{V}_{k}(4)$ is one dimensional over $\mathbf{Q}$.

In their paper, P. and S. Chowla [4] mention the following number

$$
\alpha:=\frac{1^{-2}-3^{-2}+5^{-2}-7^{-2}+-\cdots}{1^{-2}-2^{-2}+4^{-2}-5^{-2}+-\cdots} .
$$

Questions about its irrationality have been raised by A. Borel, Lichtenstein, Milnor and Thurston (see [4]). We note the following.

Proposition 5 The Chowla-Milnor conjecture for $q=12$ and $k=2$ implies that $\alpha$ is irrational.

Proof We note that

$$
\alpha=\frac{1^{-2}-3^{-2}+5^{-2}-7^{-2}+-\cdots}{1^{-2}-2^{-2}+4^{-2}-5^{-2}+-\cdots}=\frac{L\left(2, \chi_{4}\right)}{L\left(2, \chi_{3}\right)},
$$


where $\chi_{4}$ and $\chi_{3}$ are odd quadratic characters modulo 4 and 3 respectively. These characters lift to two distinct imprimitive characters modulo 12. If $\alpha$ is a rational number, then since

$$
\mathrm{L}\left(2, \chi_{4}\right)-\alpha \mathrm{L}\left(2, \chi_{3}\right)=0,
$$

this will imply that the dimension of $\mathrm{V}_{2}(12)$ is at most 3. Thus the Chowla-Milnor conjecture for $q=12, k=2$ implies the irrationality of $\alpha$.

The Chowla-Milnor conjecture suggests the following curious assertion about L-functions associated to periodic functions.

Proposition 6 Suppose that Chowla-Milnor conjecture is true. Then for any nonzero rational-valued periodic function $f$ with prime period, $\mathrm{L}(s, f)$ is holomorphic at $s=1$ implies that it does not vanish at all integers $k>1$.

Proof Let $f$ be as above and $L(k, f)=0$. Then by the conjecture of Chowla and Milnor, this implies that

$$
f(1)=f(2)=\cdots=f(p-1)=\frac{f(p)}{1-p^{k}} .
$$

But since $f$ is a non-zero function,

$$
\sum_{a=1}^{p} f(a)=\frac{f(p)(p-1)}{1-p^{k}}+f(p) \neq 0 .
$$

Thus $L(s, f)$ has a pole at $s=1$.

Finally, motivated by our investigation, we formulate a stronger version of the conjecture due to Chowla and Milnor.

Conjecture (Strong Chowla-Milnor) For any $k, q>1$, the following $\varphi(q)+1$ real numbers are linearly independent over the rational numbers:

$$
1, \zeta(k, a / q) \text { with } 1 \leq a \leq q,(a, q)=1 .
$$

For any integer $k>1$, let $\widehat{V}_{k}(q)$ be the $\mathbf{Q}$-linear space spanned by

$$
\{1, \zeta(k, a / q): 1 \leq a<q,(a, q)=1\} .
$$

Proposition 7 Let $k>1$ be an odd integer. Then the following statements are equivalent:

(i) Either $\operatorname{dim}_{\mathrm{Q}} \widehat{\mathrm{V}}_{k}(3)=3$ or $\operatorname{dim}_{\mathrm{Q}} \widehat{\mathrm{V}}_{k}(4)=3$.

(ii) The number $\zeta(k)$ is irrational. 
Proof We have

$$
\begin{gathered}
\left(3^{k}-1\right) \zeta(k)=\zeta(k, 1 / 3)+\zeta(k, 2 / 3), \\
\left(4^{k}-1\right) \zeta(k)=\zeta(k, 1 / 4)+\zeta(k, 3 / 4)+\left(2^{k}-1\right) \zeta(k) .
\end{gathered}
$$

Therefore (i) implies (ii).

Conversely, suppose that (i) is false. To begin with, we note that $D^{k-1}(\pi \cot \pi z)$ is a $\mathbf{Z}$ linear combination of sums of the form $\pi^{k}(\csc \pi z)^{2 j}(\cot \pi z)^{k-2 j}$. When $k-1$ is even, $\left.D^{k-1}(\pi \cot \pi z)\right|_{z=1 / 3}$ is a rational multiple of $\sqrt{3} \pi^{k}$, but $\left.D^{k-1}(\pi \cot \pi z)\right|_{z=1 / 4}$ is a rational multiple of $\pi^{k}$.

Then by using equation (2) in the proof of Theorem 1, we have

$$
\left(3^{k}-1\right) \zeta(k)=a \sqrt{3} \pi^{k}+b
$$

and

$$
\left(4^{k}-2^{k}\right) \zeta(k)=c \pi^{k}+d
$$

where $a, b, c, d$ are all rational numbers. Since $\pi$ is transcendental and $\sqrt{3}$ is algebraic irrational, we have $a=c=0$ and hence $\zeta(k)$ is rational.

Remark Since it has been established by Apéry that $\zeta(3)$ is irrational, we have that either $\operatorname{dim}_{\mathrm{Q}} \widehat{\mathrm{V}}_{3}(3)=3$ or $\operatorname{dim}_{\mathrm{Q}} \widehat{\mathrm{V}}_{3}(4)=3$. Further, by a result of Rivoal and Ball [2], $\zeta(k)$ is irrational for infinitely many odd $k$. Thus for infinitely many odd $k$, either $\operatorname{dim}_{\mathbf{Q}} \widehat{\mathrm{V}}_{k}(3)=3$ or $\operatorname{dim}_{\mathrm{Q}} \widehat{\mathrm{V}}_{k}(4)=3$.

Acknowledgement We are grateful to Professor Michel Waldschmidt and the referee for their helpful comments on an earlier version of our paper.

\section{References}

[1] A. Baker, B. J. Birch and E. A. Wirsing, On a problem of Chowla. J. Number Theory 5(1973), 224-236. doi:10.1016/0022-314X(73)90048-6

[2] K. Ball and T. Rivoal, Irrationalité d'une infinité de valeurs de la fonction zeta aux entiers impairs. Invent. Math. 146(2001), 193-2007. doi:10.1007/s002220100168

[3] S. Chowla, The nonexistence of nontrivial linear relations between the roots of a certain irreducible equation. J. Number Theory 2(1970), 120-123. doi:10.1016/0022-314X(70)90012-0

[4] P. Chowla and S. Chowla, On irrational numbers. Norske Vid. Selsk. Skr. (Trondheim) 3(1982), 1-5. (See also S. Chowla, Collected Papers, Vol. 3. CRM, Montreal, 1999, 1383-1387.)

[5] A. Goncharov, Multiple $\zeta$-values, Galois groups, and geometry of modular varieties. In: European Congress of Mathematics, Vol. I (Barcelona, 2000), Progr. Math. 201, Birkhäuser, Basel, 2001, 361-392.

[6] V. Kumar Murty and M. Ram Murty, Transcendental values of class group L-functions. Math Annalen, Published online, December 2010. doi:10.1007/s00208-010-0619-y

[7] - Transcendental values of class group L-functions, II. Proc. Amer. Math. Soc., to appear.

[8] S. Lang, Algebra. Revised third edition, Graduate Texts in Mathematics 211, Springer-Verlag, New York, 2002.

[9] J. Milnor, On polylogarithms, Hurwitz zeta functions, and their Kubert identities. Enseignement Math. (2) 29(1983), 281-322.

[10] H. L. Montgomery and R. C. Vaughan, Multiplicative Number Theory I: Classical Theory. Cambridge Studies in Advanced Mathematics 97, Cambridge University Press, Cambridge, 2007.

[11] M. Ram Murty and N. Saradha, Special values of the polygamma functions. Int. J. Number Theory 5(2009), 257-270. doi:10.1142/S1793042109002079 
[12] T. Okada, On an extension of a theorem of S. Chowla. Acta Arith. 38(1980/81), 341-345.

[13] T. Terasoma, Mixed Tate motives and multiple zeta values. Invent. Math. 149(2002), 339-369. doi:10.1007/s002220200218

[14] D. Zagier, Values of zeta functions and their applications. In: First European Congress of Mathematics, Vol. II (Paris, 1992), Progr. Math. 120, Birkhäuser, Basel, 1994, 497-512.

The Institute of Mathematical Sciences, CIT Campus, Taramani, Chennai 600113, India e-mail: sanoli@imsc.res.in

Department of Mathematics, Queen's University, Kingston, ON K7L 3N6, Canada

e-mail: murty@mast.queensu.ca

Chennai Mathematical Institute, Plot No H1, SIPCOT IT Park, Padur PO, Siruseri 603103, Tamil Nadu, India

e-mail: rath@cmi.ac.in 